Journal of Case Reports 2017;7(2):152-154

\title{
Pyriform Sinus Fistula Presenting as Fetal Cervical Cyst
}

\author{
Masaya Hirose, Hiroshi Sato, Saeko Imai, Hiroko Yano, Nao Taguchi, Takako Suzuki
}

Department of Obstetrics and Gynecology, Hyogo Prefectural Amagasaki General Medical Center, 2-17-77, Higashinaniwa, Amagasaki, 660-8550, Japan.

\section{Corresponding Author:}

Dr. Masaya Hirose

Email: tmhirose@hp.pref.hyogo.jp

This is an Open Access article distributed under the terms of the Creative Commons Attribution License (creativecommons.org/ licenses/by/3.0).

Received

Accepted

Published

August 29, 2016

December 14, 2016

April 25, 2017

\begin{abstract}
Background: Pyriform sinus fistula, congenital anomaly originating from the third or fourth brachial pouch remnant, rarely occurs during the prenatal period. Case Report: A pregnant woman was referred to our hospital at 30 weeks of gestation with ultrasonographic finding of fetal cervical cyst. Fetal ultrasonography had revealed a left unilocular cystic lesion in neck.. Although it remained unchanged in size, deviation of the trachea by the cyst was later demonstrated by magnetic resonance imaging. A male child was born with a good Apgar score by elective cesarean delivery. Mild respiratory dysfunction was resolved by immediate cyst aspiration. Our diagnosis was pyriform sinus fistula with subcutaneous cyst formation based on catheterization performed under laryngoscopy. Our hospital pediatric surgeons removed the cyst and fistula, and the postoperative course was uneventful. Conclusion: The possibility of pyriform sinus fisuta must be kept in mind for fetal cystic lesions of neck.
\end{abstract}

Keywords: Cysts, Pregnancy, Pyriform Sinus, Trachea, Ultrasonography.

\section{Introduction}

Pyriform sinus fistula, a congenital anomaly, originates from the third or fourth brachial pouch remnant. There is potential risk of respiratory dysfunction and thyroiditis in view of pyriform sinus fistula lying closer to airway and thyoid gland [1]. Prenatal recognition is extremely rare and limited to patients with cystic lesions [2]. In the present case, a cervical cystic lesion was identified during the prenatal period and diagnosed after birth as pyriform sinus fistula.

\section{Case Report}

A 36-year-old female, gravida 5, para 4, was referred to our hospital at 30 weeks of gestation due to a fetal cervical mass revealed by routine ultrasonography. We performed fetal ultrasonography at 32 weeks of gestation, which showed a unilocular cystic lesion in the left side of the neck sized $4.6 \times 2.6 \mathrm{~cm}$ [Fig.1]. Magnetic resonance imaging at 34 weeks of gestation showed that size of the neck cyst remained unchanged, whereas deviation of the trachea was evident [Fig.2]. Cooperation with pediatric surgeons was thought necessary for the possibility of respiratory dysfunction after birth, thus an elective cesarean delivery was conducted at 38 weeks of gestation without utilizing ex-utero intrapartum treatment (EXIT). A male baby weighing 2840 grams was born with an Apgar score of 8 at 1 minute and 9 at 5 minutes. The infant showed mild respiratory dysfunction and aspiration of the left-sided cervical mass was immediately performed [Fig.3]. Serous fluid $(58 \mathrm{ml})$ was obtained and cytologic findings indicated similarities to amniotic fluid. On the day after birth, the neck cyst, originally shrunk by aspiration, had again expanded and was filled with air [Fig.4]. A diagnosis of pyriform sinus fistula was confirmed by catheterization of the fistula as part of a preoperative laryngoscopy procedure. Our hospital pediatric surgeons removed the cyst and fistula 7 days after birth and the postsurgical course was uneventful. 

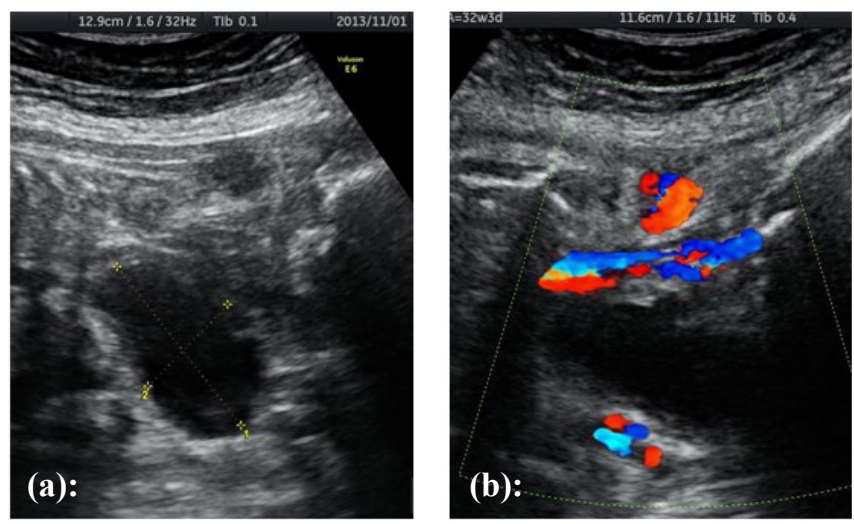

Fig.1: Fetal ultrasonography at 32 weeks of gestation. (a): monolocular cystic mass in left side of neck. (b): no blood flow in the cyst was detected.
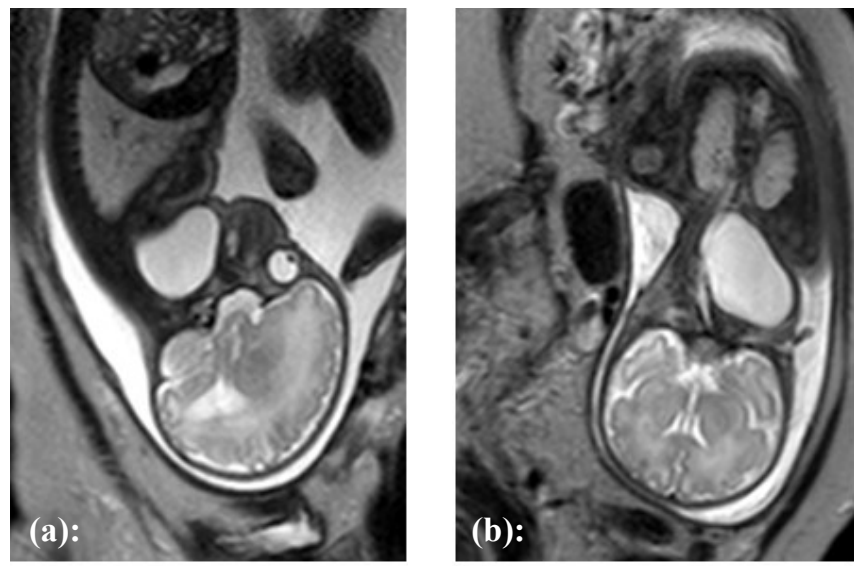

Fig.2: T2-weighted magnetic resonance imaging at 34 weeks of gestation. (a): sagittal view showing cystic lesion in anterior portion of the neck. (b): coronal view showing deviation of the trachea to right side by compression of the cyst.

\section{Discussion}

Most cases of pyriform sinus fistula are detected in childhood or older years, and rarely during the prenatal period. Kakogawa et al. [3] reported three cases of prenatal detection of pyriform sinus fistula, each of which resulted in a cystic lesion in the fetal neck. A pyriform sinus fistula in addition to congenital goiter, solid thyroid tumor, thyroid cyst, brachial cleft cyst or laryngocele should be considered for differential diagnosis of a fetal cervical cystic lesion [4].
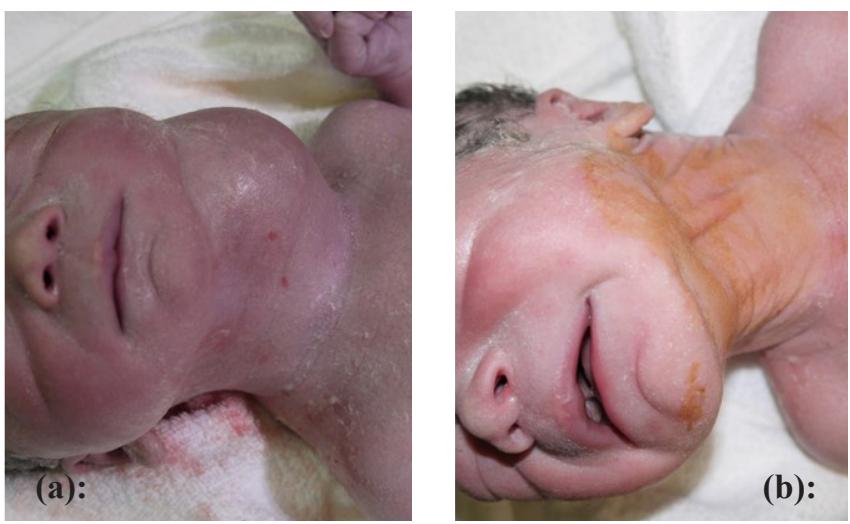

Fig.3: Depiction of neck of the newborn. (a): before aspiration. (b): after aspiration.
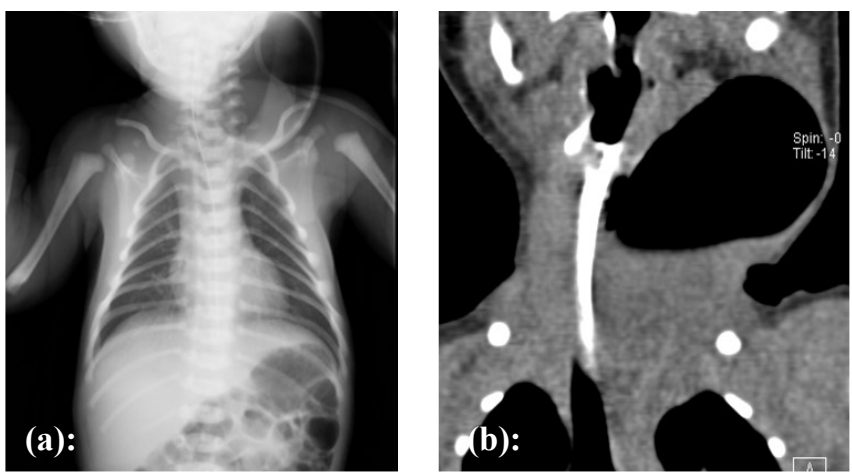

Fig.4: Neck of newborn on day one of life. (a): X-ray image. (b): CT image obtained with airway injection of contrast media.

Although a pyriform sinus fistula often causes infectious events including thyroiditis in childhood or older patients [5], those detected during the antenatal period will have cyst formation in an area near the trachea, which can cause airway obstruction. Although there are no reports of complete airway obstruction in related cases, Kakogawa et al. [3] introduced EXIT in their report. Deviation of the trachea in the present case was not so severe and we anticipated that decompression could be attained by immediate aspiration of the cyst, thus EXIT was not thought to be suitable. However, the cooperation of pediatric surgeons was needed during the elective cesarean delivery procedure, as the newborn showed mild respiratory dysfunction, which resolved by 
immediate aspiration of the cyst by the pediatric surgery team.

Fetal intervention can be considered for avoidance of cesarean delivery with or without utilizing EXIT. However, since the cyst can easily refill through the fistula, as observed in our case, the interval between intervention and delivery may be important, and further evaluation is warranted.

\section{Conclusion}

A cystic lesion detected in the neck of a fetus was diagnosed as pyriform sinus fistula after birth. Immediate aspiration of the cyst was highly effective for neonatal respiratory distress due to tracheal deviation.

Contributors: MH: Manuscript writing, case management; HS, SI: manuscript editing, case management; HY, NT, TS: critical inputs into the manuscript and literature search. $\mathrm{MH}$ will act as guarantor. All authors approved the final version of the manuscript.

Funding: None; Competing interests: None stated.

\section{References}

1. Kubota M, Suita S, Kamimura T, Zaizen Y. Surgical strategy for the treatment of piriform sinus fistula. J Pediatr Surg. 1997;32:34-37.

2. Yanai T, Yamataka A, Kobayashi H, Lane GJ, Miyano. Suspicion of prenatal pyriform sinus cyst and fistula: a case report. Pediatr Surg Int. 2004;20:58-60.

3. Kakogawa J, Nako T, Igarashi S, Nakamura S, Tanaka M. Prenatal diagnosis of piriform sinus fistula: case report and literature review. Clin Case Rep. 2015;3:106109.

4. Bianchi DW, Crombleholme TW, D’Alton ME, Malone FD. Fetology Diagnosis and management of the fetal patient, $2^{\text {nd }}$ ed. New York The McGraw-Hill Company; 2010.

5. Amano H. Differences in the characteristics and management of piriform sinus fistula between neonates and young children. Pediatr Surg Int. 2012;28:15-20. 